\title{
Aligning Data Collection with Multi-dimensional Construct Definitions: The Example of Behavioral Tasks for Measuring Risk-taking Behavior
}

\author{
Carl W. Lejuez, Dean, College of Liberal Arts \& Sciences, \\ University of Kansas
}

I n their seminal work, Jessor and Jessor (1977) defined risk-taking as "behavior that is socially defined as a problem, a source of concern, or as undesirable by the norms of conventional society and the institutions of adult authority, and its occurrence usually elicits some kind of social control response" (p. 33). Focusing more explicitly on the potential consequences or outcomes of such behavior, risk-taking has also been conceptualized as behavior that involves some potential for harm or negative consequence to the individual, but that may also result in a positive outcome or reward (Byrnes et al. 1999; Leigh 1999). Further, the propensity to take risks exists on a continuum, with some risk-taking being adaptive, and only more extreme levels being maladaptive (Bornovalova et al. 2009). The availability of well-developed behavioral methodologies used to study risk-taking behavior and to ensure quality data that are reliable, valid, and meaningful are crucial to the advancement of our understanding of the processes underlying the development and maintenance of risk behaviors (Koffamanus \& Kaplan, in press).

\section{Decision-making Risk-taking Tasks The Iowa Gambling Task}

The original gold standard measure of risk taking is the Iowa Gambling Task (IGT; Bechara et al. 1994). The IGT is a decision making task originally developed to examine decisional processes associated with neuropsychological impairment (e.g., Rogers et al. 1999a). At the beginning of the task, the participant is given $\$ 2,000$, is instructed to maximize earnings over the course of 100 decisionmaking trials, and is provided with four decks of cards on the computer screen. As described by Bechara et al. (2001), the decks are labelled A, B, C, and D at the top end of each deck. All cards are identical, and each card is associated with hypothetical payoffs or losses (although versions with real financial contingencies are available). Cards from decks A and B pay an average of $\$ 100$, but also contain cards with higher losses, while cards from decks $C$ and $D$ pay an average of $\$ 50$, but losses are smaller. Accordingly, 10 draws from decks A and B (the "disadvantageous" decks) lead to a net loss of $\$ 250$, while 10 draws from decks C and D (the "advantageous" decks) lead to a net gain of $\$ 250$ (Bechara et al. 1994; Buelow and Suhr 2009).

During the task, the participant clicks on a card from any of the four decks. Once the card is selected, the computer makes a sound similar to that of a slot machine. The selected card appears as either red or black, indicating whether money was lost or gained, and the value of the 
reward or loss appears at the top of the screen. Following this feedback, the card disappears and the participant selects another card. Each deck of cards is programmed to have 60 cards (30 red and 30 black), although the participant is unaware of how many cards of each type are in each deck. Losses are equally frequent in each deck. Several dependent variables from the IGT indicate RDM, but the most widely reported indices are the number or percentage of disadvantageous choices over 100 trials, with larger values representing greater riskiness.

While there is value in considering risk taking this way, there are concerns about the challenge in being able to repeat the task given the participants goal is to "figure out" the risky options and avoid them. Thus, once this is figured out, regardless of how long that has taken, there is no way to return that same participant to the naïve state in relation to the goals of the task. Researchers have attempted to address this issue, but it remains a challenge for studies that require repeated measures (Almy, Kuskowski, Myers, \& Luciana, in press). The nature of the task also presents a conceptual challenge as the task's focus on risky decision making means that it only provides one narrow way to explore risk taking. Indeed, understanding the maladaptive aspects of risk taking from a decision making perspective is at the same time incredibly valuable for understanding one aspect of risk taking but also too narrow to be considered a comprehensive assessment. Given that risk taking is so multidimensional, the argument is not that the IGT should be discarded given its narrowness, but instead that it should be categorized clearly for the aspects of risk taking it does represent well and there should be simultaneous efforts to develop tasks that are targeted at the other dimensions of the construct. The remainder of this paper seeks to discuss this very goal.

\section{Risk-Taking Propensity Tasks}

In considering other key dimensions of risk taking, an obvious starting point is the movement away from examining risk taking as solely maladaptive and instead as a continuum, ranging from maladaptive in different ways at its extremes and adaptive in moderation. Such an approach makes intuitive sense with one receiving few rewards when taking no risks whatsoever and too many negative consequences when extreme risks are taken, thereby leaving risk taking in moderation as an ideal goal in most situations, and the outcome measure most relevant being risk taking propensity (RTP). As our research group began to develop a task to do this very thing, we found a simple approach that had been around for some time that could serve as a starting point.

\section{Slovic's Devil Task}

The first behavioral task designed and used to assess RTP was Slovic's Devil Task (1966). Although it was not used to assess risk-taking related to substance use in the extant literature, review of the Devil Task is important for its historical significance and relation to current, commonly used RTP behavioral measures.

In the Devil Task originally implemented by Slovic (1966), participants were seated before a panel of ten small knife switches and told that nine of the switches were "safe" and that the tenth was a "disaster" switch, with it being impossible to distinguish which was the dis- 
aster switch. Each participant was informed that a switch could only be pulled once (i.e., sampled without replacement), thus the likelihood of pulling the disaster switch increased with each subsequent trial. The participant was then asked to pull one of the switches and, if the participant chose a safe switch, he/she was allowed to place one spoonful of candies into a glass bowl. The participant then had to decide whether to pull another switch or to stop and keep the candy he/she had already won. If the participant decided to continue but subsequently pulled the disaster switch, a buzzer would sound and the participant would lose everything he/she had already earned. The task ended when the participant either chose to stop and collect his/her winnings or pulled the disaster switch and lost everything. In the event that the participant pulled nine safe switches in a row, he/she was automatically forced to stop and take his/her nine spoonfuls of candy, as the only remaining switch necessarily was the "disaster" switch. Slovic (1966) argued that because both the probability and magnitude of one's potential loss increases with the number of switches pulled, stopping performance on the task can be considered an index of risk-taking tendencies. A slightly altered version of the Devil Task includes a presentation with ten wooden boxes, where one box contains a devil. The potential reward for this version of the task are stickers (Hoffrage et al. 2003). A computerized version of the Devil Task using boxes also exists (Eisenegger et al. 2010).

The Devil Task is most notable for its historical significance. Despite its limited use, the task possesses multiple features that make it a useful measure for studying risk-taking. For example, the Devil Task discriminates between risk takers without involving learning (Hoffrage et al. 2003). Although few variants of the task have been tested, the task could be a useful vehicle for examining contextual influences on risk-taking such as the impact of varying either the number of switches and/or the magnitude of stakes. Because the task has clear face validity, it is likely useful without modification across developmental stages, and the short administration time makes it ideal for examining the impact of experimental manipulations such as stress or drug/alcohol administration.

\section{Balloon Analogue Risk Task (BART)}

The Devil Task provided a simple model of RTP. This simplicity gave rise to our development of a task that took the basic idea of measuring RTP but was done in a way that would allow for increasingly complex ways to study the type of complex risk behaviour seen in the real world. This work led to the development of the Balloon Analogue Risk Task (BART; Lejuez et al. 2002).

The BART is a computerized measure of RTP that models real-world risk behavior through the conceptual framework of risky behaviors in having both the potential for reward as well as the risk of harm (Leigh 1999; Lejuez et al. 2002). In the task, the participant is presented with a balloon and asked to pump the balloon by clicking a button on the screen. With each click, the balloon inflates $.3 \mathrm{~cm}$ and money is added to the participant's temporary winnings; however, if the participant pumps up the bal- 
loon beyond its explosion point, the balloon explodes and he/she loses the money earned on that balloon. The task is depicted in the Figure below.

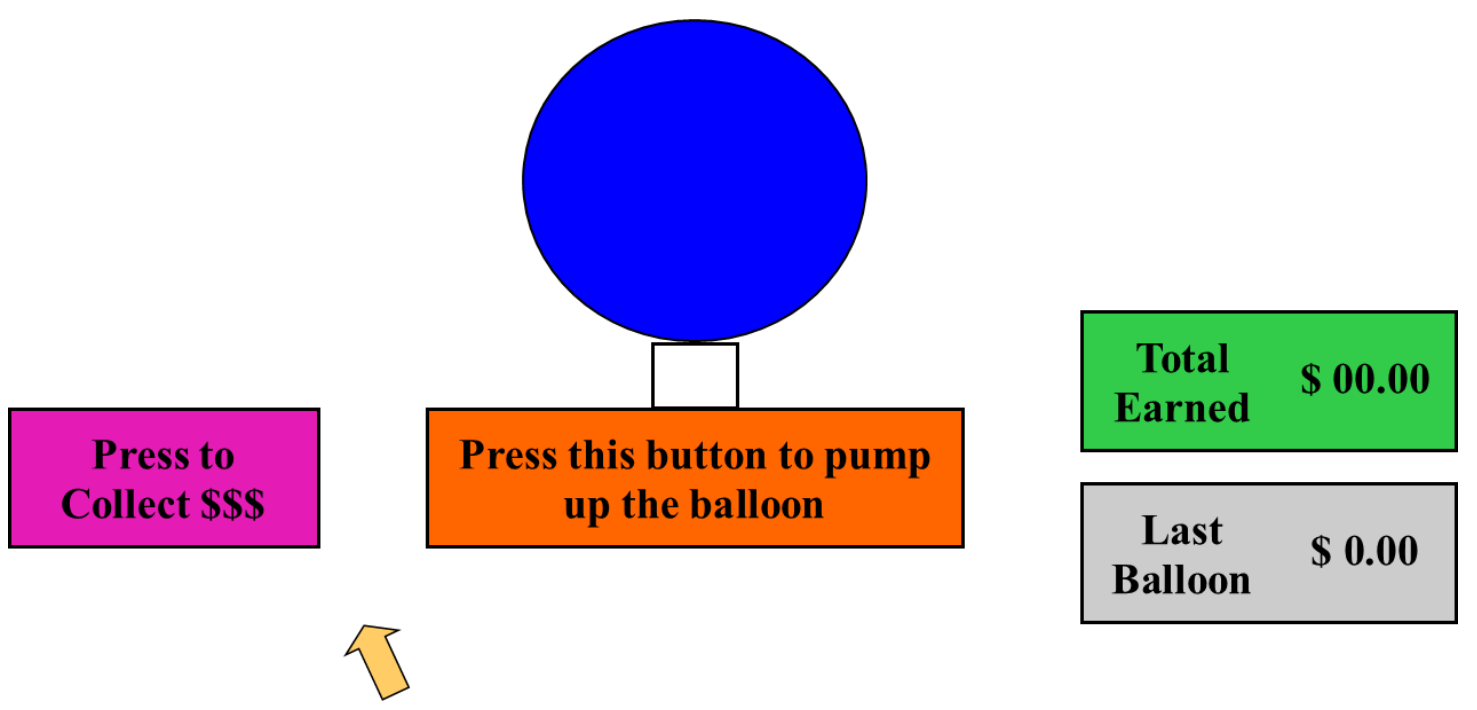

The explosion point can be varied both across and within studies. Lejuez et al. (2002) used three different balloon colors, with each color associated with a range of $1-8,1-32$, or $1-128$ pumps, respectively. The probability that a balloon would explode was arranged by constructing an array of $N$ numbers. The number 1 was designated as indicating a balloon explosion. On each pump of the balloon, a number was selected without replacement from the array. The balloon exploded if the number 1 was selected. For example, for the blue balloon ranging from 1-128, the probability that a balloon would explode on the first pump was $1 / 128$. If the balloon did not explode after the first pump, the probability that the balloon would explode was $1 / 127$ on the second pump, $1 / 126$ on the third pump, and so on up until the 128th pump, at which the probability of an explosion was $1 / 1$ (i.e., $100 \%$ ). According to this algorithm, the average break point would be the midpoint of the range, in this case 64 pumps. Before the balloon pops, the participant can press "Collect $\$ \$$ " which saves his or her earnings to a permanent bank. If the balloon pops before the participant collects the money, all earnings for that balloon are lost, and the next balloon is presented.

RTP on the BART is defined as the adjusted average number of pumps on unpopped balloons (Bornovalova et al. 2005; Lejuez et al. 2002), with higher scores indicative of greater RTP. In the original version of the task, each pump was worth $\$ .05$ and there were 30 total balloons. Participants were provided this information, but were not given information about the breakpoints. This instructional set allowed for the examination of participants' initial responses to the task and to changes as they experienced the contingencies related to payout collections and balloon explosions. Results of the original study also established 
that the relationships between key outcome variables and BART scores were most evident at the largest balloon range of 1-128, which has largely been used in subsequent studies.

The BART has well-established reliability across a range of samples. Splitthird reliability has been examined by comparing scores across the first block of 10 balloons, middle block of 10 balloons, and final block of 10 balloons on the task. The reliability estimates typically indicate strong correlations $(>0.7)$ among the blocks (Lejuez et al. 2010; Lejuez et al. 2002). Extended test-retest reliability has been indicated with the task presented twice across a two-week period, with a nonsignificant increase across administrations (T2-T1 $\Delta=1.2$ adjusted average pumps) and a reasonably robust test-retest correlation (T1/T2 $r=.77$; White et al. 2008).

It is notable that relationships of performance on the BART to self-report measures of disinhibition are inconsistent. Findings indicate modest, though significant, relationships with sensation seeking $(r=\sim .20)$, but typically nonsignificant relationships with self-report and other behavioral measures of impulsivity (Bornovalova et al. 2005; Lejuez et al. 2007; Meda et al. 2009).

The BART is considered to be one of a small number of gold-standard measures of risk-taking (Harrison et al. 2005). Within adolescent studies, RTP as measured by the BART is related to adolescent self-reported engagement in realworld risk-taking behaviors including substance use behaviors and delinquency/safety behaviors (Aklin et al. 2005; Fernie, Goudie, \& field, 2010; Lejuez et al. 2003b). Greater levels of RTP on the
BART have been found in adolescent ever-smokers as compared to neversmokers as well as in adolescents with conduct and substance problems when compared to matched controls (Crowley et al. 2006; Lejuez et al. 2005). Performance on the BART-Y (a youth version; Lejuez et al. 2007) correlates significantly with a variety of real-world risky behaviors. Specifically, greater RTP on the BART-Y is associated with increased frequency of substance use, gambling, delinquent behaviors, and risky sexual behavior (Aklin et al. 2005; Hamilton, Felton, Risco, Lejuez, \& MacPherson, 2014; Lejuez et al. 2007; Lejuez et al. 2005; Macpherson 2010).

\section{Prediction of Future Risk Behavior}

Of particular importance is the extent to which the BART can be used to predict future risk behavior. As noted above, existing data often shows a correlation between risk taking on the task and current levels of real world risk behavior, and a handful of studies have further shown that changes in risk-taking behavior in the real world over a period of time correlate with changes in risk taking on the BART over the same period of time (MacPherson et al. 2010). However, there appears to be no evidence of risk taking on the BART at one time point predicting future risk taking behavior. The absence of prediction data considered together with reasonably solid evidence of crosssectional relationships between BART suggest that while the BART may not be a task to predict who will be risky in the future, its does a competent job of serving as a proxy of existing risk behavior in the real world. Towards this end, we have completed several studies that leverage this opportunity to understand how risk 
taking is impacted by a range of external factors in the real world by manipulating those factors in a controlled laboratory environment.

Reward density and category. The BART has one of the few studies examining the effects of varying cash reward magnitudes on RTP, as a function of individual differences in impulsivity (Bornovalova et al. 2009). Specifically, Bornovalova and colleagues manipulated the magnitude of reward/loss value, examining differences in BART score at 1,5 , and 25 cents per pump, as a function of trait impulsivity and sensation seeking. As the magnitude of monetary reward/loss value increased, risk-taking on the task decreased. However, when examined separately among individuals with high impulsivity/sensation seeking as compared to individuals low in these traits, the negative relationship between reward/loss magnitude and riskiness appeared most prominent among individuals low in impulsivity/sensation seeking. Conversely, individuals high in impulsivity/sensation seeking showed little change in riskiness as reward/loss magnitude increased. These findings illustrate that higher reward/loss magnitudes convey less risktaking on the task, particularly for individuals low in impulsivity-related traits, suggesting that researchers should consider the value of reinforcers that are employed in behavioral risk-taking paradigms when interpreting results.

Peer Influence. Reynolds (2014) examined the effect of peer influence on BART RTP. Results of this study indicated that while no differences existed between the groups at baseline, RTP on the BART was significantly greater at a second experimental session for individuals who had peers encouraging their risk-taking as compared to individuals who completed the second session alone or with peers solely present, but not encouraging. In a second study, Cavalca et al. (2013) found that youth who were smokers (a proxy for higher levels of real world risk taking) were more influenced by peer pressure to be risky than youth who were not smokers. These data suggest that risk taking and the influence of peer pressure on risk taking can be assessed using the BART.

Anxiety. One particularly challenging question in the risk taking literature is the impact of anxiety. While anxiety is more generally thought to be negatively related to risk taking (e.g., Butler \& Matthews, 1987), other evidence suggests that risk behaviors increase among those with anxiety in situations in which an anxiety-inducing stimulus is present (Kashdan \& Hofmann, 2008). While this dichotomy makes intuitive sense it is difficult to test tease apart the disposition vs situation impact on risk taking of those with elevated anxiety. To address this issue, Reynolds et al. (2013) utilized an anxiety induction with a community sample of adolescents and compared performance on the BART in a high and low stress state (see the top of the Figure below for a diagram of the study). The high stress state included informing participants that they would be giving a speech at the end of the study and in the interim, they watched study confederates giving the speech and getting a difficult response from the audience; the low stress state included watching pleasant videos. Participants completed the BART before the low or high stress induction and again after the induction to examine the impact of the induction on risk taking as 
a function of anxiety level. Participants were partitioned based on their level of anxiety specific to social phobia (resulting in a high and low social phobia group). As shown in the bottom half of the Figure below, an interaction of group (low social phobia versus high social phobia) by condition was observed. Those in the high social phobia group had significantly increased risk-taking behavior from the control to the experimental session. Moreover, while the report of negative affect was similar for both groups in the low stress condition, the high social phobia group reported significantly higher self-reported distress during the high stress condition. This suggests that a task such as the BART can be useful in isolating the impact of anxiety inducing stimuli, and that for those showing greater impact of that stimuli a corresponding increase in risk taking behavior is observed.

Low Stress Session

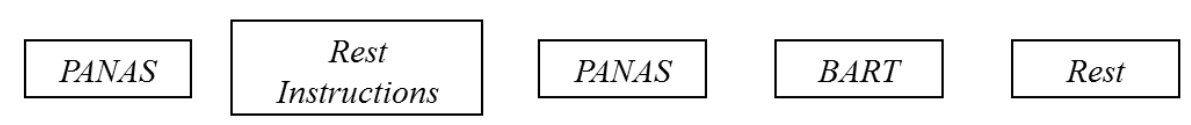

High Stress Session
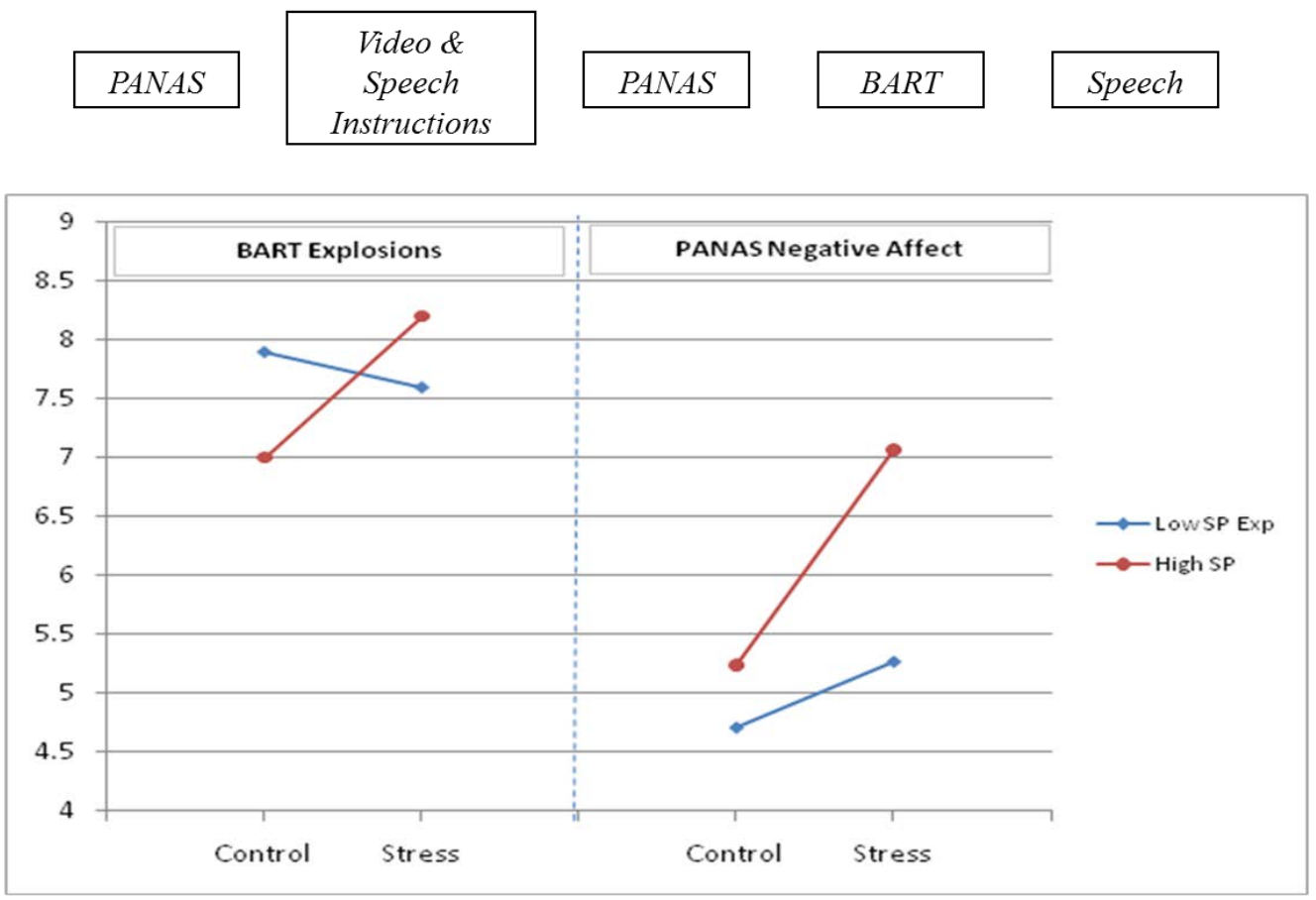


\section{Conclusion}

In conclusion, data plays an important role in our understanding of real world risk taking behavior. Ensuring the quality of that data requires an understanding of the rationale for the tasks developed and used. It also requires a clear sense of what useful existing data or new behavioral tasks can provide and where they fall short. The IGT and the BART assess risk taking in different ways, and together they may provide a strong comprehensive picture. Nonetheless, there is little data from any task suggesting strong longitudinal predictions. Thus, at least for now it seems clear that isolating risk taking in a controlled laboratory setting and providing the opportunity to manipulate key variables thought to impact risk behavior in the real world should be the focus on experimental studies. We have begun to conduct this work with the BART and have reviewed some early studies above, but considerable work including studies that bring in genetic factors and neural assessment (Bjork \& Pardini, 2015; Gu, Zhang, Luo, Wang, \& Broster, in press) as well as a wider range of environmental factors will be crucial to any further progress.

\section{References}

Aklin, W. M., Lejuez, C. W., Zvolensky, M. J., Kahler, C. W., \& Gwadz, M. (2005). Evaluation of behavioral measures of risk taking propensity with inner city adolescents. Behavior Research and Therapy, 43, 215-228.

Amy, B., Kieslowski, M., Malone, S. M., Myers, E., \& Luciana, M. (in press). A longitudinal analysis of adolescent decision-making with the Iowa Gambling Task. Developmental Psychology.

Bechara, A., Damasio, A. R., Damasio H., \& Anderson, S. W. (1994). Insensitivity to future consequences following damage to human prefrontal cortex. Cognition, 50, 7-15.

Bechara, A., Dolan, S., Denburg, N., Hindes, A., Anderson, S. W., \& Nathan P . E. (2001). Decision making deficits, linked to a dysfunctional ventromedial prefrontal cortex, revealed in alcohol and stimulant abusers. Neuropsychologia, 39, 376-389.

Bjork, J. M., \& Padroni, D. A. (2015). Who are those risk-taking adolescents?: Individual differences in developmental neuroimaging research. Developmental Cognitive Neuroscience, 11, 56-64.

Bornovalova, M. A., Cashman-Rolls, A., O'Donnell, J. M., Ettinger, K., Richards, J. B., Dewit, H., \& Lejuez, C. W. (2009). Risk taking differences on a behavioral task as a function of potential reward/loss magnitude and individual differences in impulsivity and sensation 
seeking. Pharmacology, Biochemistry, and Behavior, 93, 258262.

Bornovalova, M. A., Daughters, S. B., Hernandez, G. D., Richards, J. B., \& Lejuez, C. W. (2005). Differences in impulsivity and risk-taking propensity between primary users of crack cocaine and primary users of heroin in a residential substance-use program. Experimental and Clinical Psychopharmacology, 13, 311-318.

Buelow, M. T., \& Suhr, J. A. (2009). Construct validity of the Iowa gambling task. Neuropsychology Review, 19, 102-114.

Butler, G., \& Mathews A. (1987). Anticipatory anxiety and risk perception. Cognitive Therapy and Research, 11, 551-565.

Byrnes, J. P., Miller, D. C., \& Schafer, W. D. (1999). Gender differences in risk taking: A meta-analysis. Psychol Bulletin, 125, 367-383.

Cavalca, E., Liss, T., Lejuez, C. W., Reynolds, E. K., Schepis, T. S., Kong, G., \& Krishnan-Sarin, S. (2013). A preliminary experimental investigation of peer influence on risk taking among adolescent smokers and non-smokers. Drug and Alcohol Dependence, 129, 163-166.

Crowley, T. J., Raymond, K. M., Mikulich-Gilbertson, S. K., Thompson, L. L., \& Lejuez, C. W. (2006). A Risk-Taking "Set" in a Novel Task Among Adolescents With Serious Conduct and Substance Problems. Journal of the American Academy of Child and Adolescent Psychiatry, 45, 175-183.
Eisenegger, C., Knoch, D., Ebstein, R. P., Gianotti, L. R R., Sándor, P. S., \& Fehr, E. (2010). Dopamine receptor D4 polymorphism predicts the effect of L-DOPA on gambling behavior. Biological Psychiatry, 67, 702-706.

Fernie, G., Cole, J. C., Goudie, A. J., \& Field, M. (2010). Risk-taking but not response inhibition or delay discounting predict alcohol consumption in social drinkers. Drug and Alcohol Dependence, 112, 54-61.

Gu, R., Zhang, D., Luo, Y., Wang, H., \& Broster, L. S. (in press). Predicting risk decisions in a modified balloon analogue risk task: Conventional and single-trial erp analyses. Cognitive, Affective \& Behavioral Neuroscience.

Hamilton, K. R., Felton, J. W., Risco, C. M., Lejuez, C. W., MacPherson, L. (2014). Brief report: The interaction of impulsivity with risk-taking is associated with early alcohol use initiation. Journal of Adolescence, 37, 1253-1256.

Harrison, J. D., Young, J. M., Butow, P., Salkeld, G., Solomon, M. J. (2005). Is it worth the risk? A systematic review of instruments that measure risk propensity for use in the health setting. Social Science $\mathcal{E}$ Medicine, 60, 1385-1396.

Hoffrage, U., Weber, A., Hertwig, R., \& Chase, V. M. (2003). How to Keep Children Safe in Traffic: Find the Daredevils Early. Journal of Experimental Psychology: Applied, 9, 249-260.

Jessor, R., \& Jessor, S. L. (1977). Problem behavior and psychosocial development: A longitudinal 
study of youth. Academic Press New York

Kashdan, T. B., \& Hofmann S. G. (2008). The high-novelty-seeking, impulsive subtype of generalized social anxiety disorder. Depression and Anxiety, 25, 535-541.

Koffarnus, M. N., \& Kaplan, B. A. (in press). Clinical models of decision making in addiction. Pharmacology, Biochemistry and Behavior,

https://www.ncbi.nlm.nih.gov/pu bmed/28851586

Leigh, B. C. (1999). Peril, chance, adventure: concepts of risk, alcohol use and risky behavior in young adults. Addiction, 94, 371383.

Lejuez, C. W., Aklin, W. M., Daughters, S. B., Zvolensky, M. J., Kahler, C. W., \& Gwadz, M. (2007). Reliability and validity of the youth version of the Balloon Analogue Risk Task (BART- Y) in the assessment of risk-taking behavior among inner-city adolescents. Journal of Clinical Child and Adolescent Psychology, 36, 106-111.

Lejuez, C. W., Aklin, W. M., Bornovalova, M. A., \& Moolchan, E. (2005). Differences in risk taking propensity across inner-city adolescent everand never-smokers. Nicotine and Tobacco Research, 7, 71-79.

Lejuez, C. W., Aklin, W. M., Jones, H. A., Richards, J. R., Strong, D. R., Kahler, C. W., \& Read, J. P. (2003). The Balloon Analogue Risk Task Differentiates smokers and nonsmokers. Experimental and Clinical Psychopharmacology, 11, 26-33.
Lejuez C. W., Aklin, W. M., Zvolensky, M. J., \& Pedulla C. M. (2003b). Evaluation of the Balloon Analogue Risk Task (BART) as a predictor of adolescent real-world risk-taking behaviours. Journal of Adolescne, 26, 475-479.

Lejuez, C. W., Read, J. P., Kahler, C. W., Richards, J. B., Ramsey, S. E., Stuart, G. L., Strong, D. R., \& Brown, R. A. (2002). Evaluation of a behavioral measure of risk taking: The Balloon Analogue Risk Task (BART). Journal of Experimntal Psychology: Applied, 8, 75-84.

Lighthall, N. R., Mather, M., \& Gorlick, M. A. (2009). Acute Stress Increases Sex Differences in Risk Seeking in the Balloon Analogue Risk Task. PLoS One, 4, e6002.

MacPherson, L., Magidson, J. F., Reynolds, E. K., Kahler, C. W., \& Lejuez, C. W. (2010). Changes in Sensation Seeking and Risk Taking Propensity Predict Increases in Alcohol Use Among Early Adolescents. Alcohol: Clinical and Experimental Research, 34, 1400-1408.

Meda, S. A., Stevens, M. C., Potenza, M. N., Pittman, B., Gueorguieva, R., Andrews, M. M., Thomas, A. D., Muska, C., Hylton, J. L., \& Pearlson, G. D. (2009). Investigating the behavioral and self-report constructs of impulsivity domains using principal component analysis. Behavioral Pharmacology, 20, 390399. 
Reynolds, E. K., Schreiber, W. M., Geisel, K., MacPherson, L., Ernst, M., \& Lejuez, C. W. (2013). Influence of social stress on risk-taking behavior in adolescents. Journal of Anxiety Disorders, 27, 272-277.

Reynolds, E. K., MacPherson, L., Schwartz, S., Fox, N. A., \& Lejuez, C. W. (2014). Analogue study of peer influence on risk-taking behavior in older adolescents. Prevention Science, 15, 842-849.

Rogers, R., Everitt, B., Baldacchino, A., Blackshaw, A., Swainson, R., Wynne, K., Baker, N., Hunter, J., Carthy, T., \& Booker, E. (1999a). Dissociable deficits in the decision making cognition of chronic amphetamine abusers, opiate abusers, patients with focal damage to prefrontal cortex, and tryptophan-depleted normal volunteers: evidence for monoaminergic mechanisms. Neuropsychopharmacology, 20, 322339.

Schepis, T. S., McFetridge, A., Chaplin, T. M., Sinha, R., \& Krishnan-Sarin, S. (2011). A Pilot Examination of Stress-Related Changes in Impulsivity and Risk Taking as Related to Smoking Status and Cessation Outcome in Adolescents. Nicotine and Tobacco Research, 13, 611-615.

Slovic, P. (1966). Risk-taking in children: Age and sex differences. Child Development, 37, 169-176.

White, T. L., Lejuez, C. W., \& de Wit, H. (2008) Test-retest characteristics of the Balloon Analogue Risk Task (BART). Experimental and Clinical Psychopharmacology, 16, 565-570. 